\title{
Cumulative social risk and type 2 diabetes in US adults: The National Health and Nutrition Examination Survey (NHANES) 1999-2006
}

\author{
Justin B Echouffo-Tcheugui ${ }^{1,2}$, Rishi Caleyachetty ${ }^{3}$, \\ Peter A Muennig ${ }^{3}$, KM Narayan' and \\ Sherita H Golden ${ }^{4,5}$
}

\begin{abstract}
Background: The cumulative effects of adverse social factors on the diabetes risk remains to be clarified.

Design: Cross-sectional analysis of the US National Health and Nutrition Examination Survey (NHANES) $1999-2006$. Methods: We included 10,276 adults aged $\geq 20$ years. Diabetes mellitus was defined by physician diagnosis or fasting plasma glucose $(\geq 126 \mathrm{mg} / \mathrm{dl})$ or glycated hemoglobin $(\geq 6.5 \%)$. Social risk factors (low family income, low education level, minority racial/ethnic group status, and single-living status) and health-related behaviors (physical activity and dietary intake) were self-reported. Social risk factors were combined in a cumulative social risk index (range 0 to $\geq 3$ ) and logistic regression used to assess the association of cumulative social risk and diabetes, taking into account complex survey design and sampling weights.

Results: Of 10,276 participants, 1515 (weighted proportion - 10\%) had diabetes, 3295 (32.3\%) and 1830 (9.0\%) were exposed to $\geq \mathrm{I}$ adverse social risk factor and $\geq 3$ social risk factors, respectively. Diabetes was associated with increasing cumulative social risk in a graded manner ( $p$ for trend $<0.00 \mathrm{I}$ ). Compared with a cumulative social risk score of 0 , the age- and sex-adjusted diabetes odds for a cumulative social risk score of $\geq 3$ was 2.84 (95\% confidence interval: $2.23-$ 3.62), and 2.72 (95\% confidence interval: 2.05-3.60) after further adjustment for family history of diabetes, body mass index, smoking, dietary intake and leisure time physical activity. Health behaviors and adiposity only partially influenced the cumulative social risk and diabetes relationship.

Conclusions: Simultaneous exposure to several adverse social risk factors significantly influences the odds of diabetes. Better prevention and control of diabetes needs accounting for all aspects of social disadvantage.
\end{abstract}

\section{Keywords}

Social risk factor, cumulative risk, diabetes, health disparities

Received 22 June 2015; accepted 23 December 2015

\section{Introduction}

Diabetes is a major public health problem in the USA, affecting $9.3 \%$ of Americans. ${ }^{1}$ Additionally, $37 \%$ of US adults have prediabetes and are therefore at increased risk for developing type 2 diabetes (T2DM). ${ }^{1}$ About one in three American adults will have diabetes by the year 2050 if current trends continue. ${ }^{2}$ Prevention and control of diabetes are therefore an urgent priority, thus requiring a more robust understanding of disease determinants. Clarifying the role of socio-environmental determinants of diabetes is of importance for

\footnotetext{
'Department of Global Health, Rollins School of Public Health, Emory University, Atlanta, USA

${ }^{2}$ Department of Medicine, Brigham and Women's Hospital, Boston, USA ${ }^{3}$ Department of Health Policy and Management, Mailman School of Public Health, Columbia University, New York, USA

${ }^{4}$ Department of Medicine, Johns Hopkins University School of Medicine, Baltimore, USA

${ }^{5}$ Department of Epidemiology, Bloomberg School of Public Health, Johns Hopkins University, Baltimore, USA
}

Corresponding author:

Justin B Echouffo-Tcheugui, Department of Global Health, Rollins School of Public Health, Emory University, I5I8 Clifton Road NE, Atlanta, GA 30322, USA.

Email: jechouf@emory.edu 
designing appropriate socially- and culturally-adapted prevention and control interventions. ${ }^{3}$ Extant evidence indicates that T2DM and related complications disproportionally affect disadvantaged social groups. Low income, ${ }^{4}$ low level of education, ${ }^{4,5}$ membership in a racial/ethnic minority group, ${ }^{6,7}$ and single-living status $^{8,9}$ have been related to diabetes. Indeed, the Eight Americas study demonstrated that health disparities can hardly be explained by race alone. ${ }^{10}$ Hitherto, studies of social determinants of diabetes have seldom accounted for the simultaneous or cumulative effects of social risk factors. For example, exploration of the cumulative effect of socio-economic position (SEP) over the life-course has typically been done by summing the number of times participants experienced a disadvantaged socio-economic situation in SEP indices representing the accumulation of these exposures. ${ }^{11,12}$ Individuals often experience marked social disadvantage in adult life from multiple types of social risk factors including not only socio-economic deprivation (e.g. low family income, low education level), ${ }^{13,14}$ but also racial discrimination and social isolation/low level of social support. ${ }^{15}$ Overlooking the cumulative effect of various social risk factors in conveying diabetes risk fails to fully capture the real-world experience of social disadvantage and may poorly inform approaches to addressing social disparities in diabetes. A clearer and better understanding of pathways through which the accumulation of various social risk factors may influence diabetes occurrence and progression may provide alternative approaches for addressing social disparities in cardiometabolic health.

Using data from the National Health and Nutrition Examination Survey (NHANES), a large US nationally representative study, we examined the cross-sectional association between cumulative social risk (CSR) and diabetes, as well as the influence of potential intermediate factors, namely smoking, diet quality, physical activity, and body mass index on this putative relationship.

\section{Methods}

\section{Study population}

NHANES is a population-based survey conducted among non-institutionalized US civilians, selected in a stratified, multistage, and probability cluster manner. ${ }^{16}$ We used the waves of NHANES conducted between 1999 and 2006. As previously described, after a household interview, participants underwent various examinations, and blood sample collection. ${ }^{16}$ We restricted our analysis to participants aged 20 years and above. All participants gave written informed consent before participation. The NHANES 1999-2006 study protocol was approved by the National Center for Health
Statistics (NCHS) of the Centers for Disease Control and Prevention.

\section{Measurements}

Individual social risk factors. Income was assessed by using the poverty income ratio, which is the ratio of the midpoint of observed family income category to the official poverty threshold (scaled to family size), published annually by the US Census Bureau (Series P-60). We dichotomized the poverty income ratio into below 1.00 (below the official definition of poverty) and 1.00 or greater (income above the poverty level). Education level was recorded as the number of years of education attended and completed, and then categorized into and then categorized into low education level $(<12$ years; representing less than high school) and high education level ( $\geq 12$ years; representing high school graduate/ General Educational Development (GED) or equivalent, some college or Associate degree (AA) degree, college graduate or above). Racial/ethnic group (a proxy for assumed race-related social structural experiences, like race discrimination) was defined by self-report as non-Hispanic White, non-Hispanic Black, MexicanAmerican or Other. Race/ethnic group was classified into two categories: minority racial/ethnic group (nonHispanic Black, Mexican-American and Other) and non-Hispanic White. Single-living status (a proxy for social isolation and low level of social support) was classified into two groups: married/living as married, or persons widowed, divorced, separated or never married.

Cumulative social risk index. Each of the four social risk factors low family income, low education level, minority race/ethnic group status, and single-living status was assigned a score of 1 for its presence and 0 for its absence. A CSR index, indicating the clustering of these factors in an individual, was created by summing the score for each variable, with the resultant overall score ranging from 0 to 4 , with higher scores indicating a higher level of cumulative social risk. People with a score of 3 or 4 were combined into one category for analyses due to small numbers in each of these categories $(n=1582$ and $n=412$ for categories 3 and 4 , respectively), resulting in the CSR index ranging from 0 to $\geq 3$. The variance inflation factor (VIF) for social risk factors were $<1.18$, indicating that multicollinearity was not an issue.

Glycemic status definition. During the home interview, participants were asked if they had ever been told by a doctor or other health professional that they had diabetes (other than during pregnancy). On the basis of this question, participants were classified as having diagnosed diabetes. Individuals without self-reported diabetes were diagnosed with diabetes if any of the 
following thresholds were exceeded: fasting plasma glucose $\geq 126 \mathrm{mg} / \mathrm{dl}$ and/or glycated hemoglobin $\left(\mathrm{HbA}_{1 \mathrm{C}}\right)$ $\geq 6.5 \% .^{17}$

Covariates. Covariates included age, sex and potential intermediate factors; the latter included smoking status, physical activity, the quality of dietary intake, and adiposity. Participants who reported currently smoking cigarettes, cigars or pipes were considered current smokers. Diet quality was measured by the Healthy Eating Index (HEI) derived from 24 hour recall. ${ }^{17}$ The total HEI score (range $0-100$ ) is calculated based on conforming to Food Guide Pyramid recommendations, as well as recommendations for total fat, saturated fat, sodium, cholesterol and variety. Each of the 10 HEI component scores contributes from 0 to 10 points to the total HEI score. ${ }^{17}$ The HEI component scores include the following measures: (i) dairy; (ii) fruit; (iii) grain; (iv) meat; (v) vegetables; (vi) total fat; (vii) saturated fat; (viii) sodium; (ix) cholesterol; and (x) variety. Diet quality was dichotomized into poor (i.e. a HEI score $<50$ ) and not poor (i.e. a HEI score $\geq 50$ ). ${ }^{18}$ Leisure time physical activity levels during the previous 30 days were assessed by questionnaire. Blood pressure, body weight and height were measured using standardized procedures; and body mass index (BMI) was derived as weight $(\mathrm{kg})$ divided by height $(\mathrm{m})$ squared. Obesity was defined as $\mathrm{BMI} \geq 30 \mathrm{~kg} / \mathrm{m}^{2}$. Lipid fractions, except low-density lipoprotein (LDL)-cholesterol levels, were measured using a Hitachi 704 Analyzer (Boehringer Mannheim Diagnostics, Indianapolis, Indiana). Hemoglobin was measured using a Bio-Rad Diamant ion exchange high-performance liquid chromatography system.

\section{Statistical analysis}

Of the 20,311 participants aged 20 years and above, we excluded those who had missing data on prevalent diabetes $(n=5)$. We further excluded participants with missing data on the social risk factors of interest (poverty index ratio $(n=1750)$, educational level $(n=31)$, and single-living $(n=471)$ and other relevant covariates including BMI, smoking, family history of diabetes, healthy eating intake and physical activity $(n=7328)$. After these exclusions, the final sample included 10,726 participants. Those excluded due to missing information were less likely to be male $(42.3 \%$ vs. $49.9 \% ; p<0.001)$ and more likely to have low family income $(21.0 \% \mathrm{vs}$. $17.9 \% ; p<0.001)$, belong to a minority race/ethnic group (54.6\% vs. $48.5 \% ; p<0.001)$, have low education (37.8\% vs. $31.4 \% ; p<0.001)$, and to live as a single person $(33.4 .8 \%$ vs. $26.8 \% ; p<0.001)$.

Baseline demographic, socioeconomic, behavioral, and biochemical characteristics were compared by diabetes status using Student's $t$-test for continuous variables and Chi-square tests for categorical variables. Continuous variables were presented as mean (standard deviation (SD), and categorical variables as percentages.

Logistic regression models were used to estimate odd ratios (ORs) for the association between social risk factors and diabetes risk. We first examined the association of each individual social risk factor of interest (low family income, low educational level, minority racial/ethnic group status, and single-living status) with diabetes, accounting for other social risk factors. Regarding the cumulative social risk score, and its relation to diabetes risk, we built sequential models, to estimate ORs for diabetes by levels of the combined score of adverse sociodemographic characteristics (CSR index of 0 was the reference group). We first adjusted for age, sex, and family history of diabetes. Then, to evaluate potential intermediate pathways by which clustering of social risk factors might lead to diabetes, we additionally adjusted for BMI, smoking status, physical activity levels and the quality of dietary intake. Linear dose-dependent associations between the CSR index and diabetes status were examined by including the index in models as a continuous variable and performing a linear trend test.

A two-sided $p$-value of 0.05 was used to define statistical significance for all analyses

All analyses were done using complex survey methods to account for the clustered design (sampling and weighting processes) with the Stata software, version 13.0 (StataCorp LP, College Station, TX, USA).

\section{Results}

Table 1 summarizes the baseline characteristics of participants by diabetes status. Among the 10,276 participants with complete data, $10 \% \quad(n=1515)$ of individuals had diabetes. The latter group was older, less physically active, more likely to be obese, less likely to be smokers, and had a better quality dietary intake than those without diabetes. Not surprisingly, individuals with diabetes had higher fasting plasma glucose, glycated hemoglobin, and total cholesterol. A weighted total of $32.3 \%$ of participants reported at least one social risk factor; $9.0 \%$ reported three or more.

As shown in Table 2, after adjustment for age, sex and family history of diabetes, people with low educational level $(\mathrm{OR}=1.51,95 \%$ confidence interval $(\mathrm{CI})$ : $1.30-1.75)$ or belonging to a minority racial/ethnic group (OR: 1.82, 95\% CI: 1.57-2.12) had a higher odds of diabetes. However, low family income $(\mathrm{OR}=1.24, \quad 95 \% \quad \mathrm{CI}: \quad 0.98-1.58)$ and single-living status (OR $=1.03,95 \%$ CI: $0.84-1.25)$ were not related 
Table I. Characteristics of participants to the National Health and Nutrition Examination Survey 1999-2006.

\begin{tabular}{|c|c|c|c|}
\hline & Diabetes & No diabetes & $p$-value \\
\hline Variables $^{\mathrm{a}}$ & $N=1515$ & $N=9211$ & \\
\hline Age, years & $58.5(0.5)$ & $44.9(0.3)$ & $<0.0001$ \\
\hline Male sex, $n(\%)$ & $760(49.8)$ & $4589(48.6)$ & 0.36 \\
\hline Smoking, $n$ (\%) & $417(19.0)$ & $3507(25.2)$ & $<0.0001$ \\
\hline Diet quality index - Healthy Eating Index & $49.0(0.4)$ & $50.4(0.2)$ & 0.0007 \\
\hline \multicolumn{4}{|l|}{ Physical activity levels } \\
\hline $0 \mathrm{~min} /$ week, $n$ (\%) & $851(50.2)$ & $3782(34.0)$ & $<0.0001$ \\
\hline I-I49 min/week, n (\%) & $290(20.0)$ & $2040(24.2)$ & $<0.0001$ \\
\hline$\geq 150 \mathrm{~min} /$ week, $n(\%)$ & 29.81 (374) & $3389(41.9)$ & $<0.0001$ \\
\hline Body mass index, $\mathrm{kg} / \mathrm{m}^{2}$ & $32.0(0.3)$ & $27.7(0.1)$ & $<0.0001$ \\
\hline Waist circumference, cm & $107.9(0.7)$ & $95.3(0.2)$ & $<0.0001$ \\
\hline Fasting plasma glucose, mg/dl & I60.1 (3.4) & $95.3(0.3)$ & $<0.0001$ \\
\hline Hemoglobin $A_{I C}, \%$ & $7.2(0.065)$ & $5.3(0.009)$ & $<0.0001$ \\
\hline Total cholesterol, mg/dl & $206.9(1.9)$ & $202.1(0.7)$ & 0.05 \\
\hline \multicolumn{4}{|l|}{ Individual social risk factors } \\
\hline Low family income, $n$ (\%) & $332(16.7)$ & $1588(13.0)$ & 0.012 \\
\hline Low education level, $n(\%)$ & $703(33.3)$ & $2667(17.9)$ & $<0.0001$ \\
\hline Minority race/ethnicity (not White), n (\%) & $888(34.3)$ & $4318(26.3)$ & $<0.0001$ \\
\hline Persons living alone, $n(\%)$ & $597(37.2)$ & $3469(34.9)$ & 0.22 \\
\hline \multicolumn{4}{|l|}{ Cumulative social risk index } \\
\hline $0, n(\%)$ & $26 I(3 \mid .3)$ & $2664(43.0)$ & $<0.0001$ \\
\hline $\mathrm{I}, \mathrm{n}(\%)$ & $466(34.3)$ & $2829(32.2)$ & $<0.000$ I \\
\hline $2, n(\%)$ & $418(20.2)$ & $2258(16.6)$ & $<0.0001$ \\
\hline$\geq 3, n(\%)$ & $370(14.3)$ & $1460(8.4)$ & $<0.0001$ \\
\hline
\end{tabular}

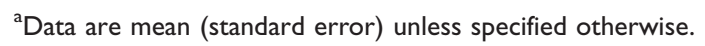

Table 2. Relationship between social risk factors and diabetes in the National Health and Nutrition Examination Survey $1999-2006$.

\begin{tabular}{|c|c|c|c|c|}
\hline \multirow[b]{2}{*}{ Single social risk factors } & \multicolumn{4}{|c|}{ Odds ratio ( $95 \%$ confidence interval) } \\
\hline & Model I & Model 2 & Model 3 & Model 4 \\
\hline Low family income & $1.25(1.01-1.55)$ & $1.24(0.98-1.58)$ & $1.21(0.96-\mid .52)$ & I. $18(0.94-1.48)$ \\
\hline Low education level & $1.50(1.29-1.74)$ & I.5I (I.30-I.75) & $1.53(1.33-1.76)$ & $1.49(1.28-1.73)$ \\
\hline Minority ethnic group & $1.85(1.59-2.16)$ & $1.82(1.57-2.12)$ & $1.82(1.56-2.12)$ & $1.81(1.55-2.12)$ \\
\hline Persons living alone & $1.016(0.84-1.23)$ & $\mathrm{I} .03(0.84-\mathrm{I} .25)$ & $\mathrm{I} .06(0.87-\mathrm{I} .29)$ & I.06 (0.87-I.28) \\
\hline \multicolumn{5}{|c|}{ Combination of social risk factors } \\
\hline \multicolumn{5}{|c|}{ Cumulative social risk index } \\
\hline I & $1.53(1.28-1.82)$ & $1.47(1.23-1.76)$ & $1.51(1.25-1.82)$ & $1.49(1.23-1.80)$ \\
\hline 2 & $2.02(1.48-2.76)$ & $2.02(1.49-2.75)$ & $2.02(1.50-2.73)$ & $1.95(1.43-2.64)$ \\
\hline \multirow[t]{2}{*}{$\geq 3$} & $2.84(2.23-3.62)$ & $2.75(2.15-3.53)$ & $2.83(2.17-3.68)$ & $2.72(2.05-3.60)$ \\
\hline & $p$ of trend $<0.001$ & $p$ of trend $<0.001$ & $p$ of trend $<0.001$ & $p$ of trend $<0.001$ \\
\hline
\end{tabular}

Model I: adjusted for age and sex; Model 2: Model I + family history; Model 3: Model 2 + body mass index; Model 4: Model 3 + smoking status, dietary intake, and leisure time physical activity.

to diabetes. Further adjustment for BMI, smoking status, dietary intake and leisure time physical activity, which can potentially be on the pathway from social risk factors to T2DM, did not change the magnitude and significance of the associations (Table 2). The direction and magnitude of these associations were not influenced by the simultaneous adjustment for other social risk factors (data not shown). 
Examination of the cumulative effect of these social risk factors (Table 2), after adjustment for age, sex and family history, revealed an increased odds of diabetes across levels of CSR compared with the reference level $(\mathrm{CSR}=0)$. Further adjustment for BMI and potential mediators including health behaviors (smoking status, dietary intake, and leisure time physical activity) had a minimal effect on the association of each level of CSR and diabetes (Table 2). Compared with those without exposure to any social risk, participants exposed to $\geq 3$ social risk factors had the highest odds of diabetes $(\mathrm{OR}=2.72,95 \%$ CI: 2.05-3.60). Additional control for BMI, smoking status, dietary intake, and leisure time physical activity, which can mediate the association of CSR and T2DM, did not change the magnitude and significance of the associations (Table 2). There was a statistically significant linear trend in the association between an increasing number of social risk factors and odds of diabetes ( $p$ for trend $<0.001$ ). For each one-point increase in the CSR score, the OR for diabetes was 1.39 (95\% CI: 1.26-1.53).

\section{Discussion}

Our findings suggest that an accumulation of social risk factors most likely has a more significant influence on the odds of diabetes than social risk factors taken individually. Indeed, adults exposed to three or more social risk factors had close to three-fold increased odds of T2DM, even after accounting for potential confounders or components of the causal pathways linking social risk factors to diabetes, such as obesity, physical inactivity, and dietary intake.

To our knowledge, this is one of the few studies that examines the cumulative influence of adverse social factors on the odds of T2DM, hence accounting for a broader exposure that captures various aspects of social disadvantage. This report also extends previous findings by highlighting the salience of cumulative social risk exposure. There may be an overlap between individual social risk factors, ${ }^{19}$ probably reflecting a broader "global social risk factor" such as a social disadvantage. Previous studies seldom accounted for the clustering of social risk factors in relation to diabetes. Studies typically examined the cumulative effect of social disadvantage by summing the effect of a single measure (e.g. manual occupational class or education) or a composite of socio-economic status assessed at different periods of the participants' lifespan. ${ }^{12,20,21}$ While these studies provided an important life-course perspective, only using lower socio-economic status as a proxy for cumulative social disadvantage fails to capture all aspects of social disadvantage or socioeconomic exposures on the risk for diabetes. Given that socio-economic deprivation may cluster with other types of social disadvantage such as racial discrimination and social isolation or low level of social support, our cumulative social risk measure may better capture the totality of social disadvantage.

\section{Potential mechanisms}

The biological pathways through which an accumulation of social risk factors would lead to diabetes remain unclear. Exposure to social stressors is posited to increase diabetes risk through behavioral factors and activation of the hypothalamic-pituitary-adrenal (HPA) axis; however, few empirical studies have rigorously tested these pathways in the development of diabetes. ${ }^{22}$ It is hypothesized that social stressors adversely affect health behaviors, such as diet, physical activity, smoking, and alcohol use, partly accounting for the high rates of obesity and diabetes seen among minorities and lower social status populations who largely experience a greater number of stressors than non-minority populations. For example, eating more in response to stress was found to partially explain the relationship between history of violence experience and obesity. ${ }^{23}$ Another proposed mechanism linking social stress and obesity/diabetes is a direct stress response through activation of the HPA axis, which in response to chronic stress has been associated with the dysregulation of cortisol. ${ }^{24}$ In turn, HPA axis dysfunction has been associated with both obesity and diabetes. ${ }^{22,25}$ Epigenetic modifications, heritable and potentially modifiable markers that regulate gene expression without changing the underlying DNA sequence, may explain the mechanisms underlying social determinants of diabetes, as epigenetic markers are responsive to non-biological and environmental exposures, particularly those encountered in early life. ${ }^{26-28}$ Indeed, existing evidence suggest an association of socio-economic circumstances with global and genome-wide DNA methylation. ${ }^{29-31}$ Understanding how social factors combine with biological pathways that lead to diabetes can inform translational research. Indeed, this can help to identify targets for change in intervention studies (e.g. decreasing social stressors at individual or neighborhood levels) or identify points for interventions (e.g. improving healthcare resources for individuals or neighborhoods experiencing high levels of social stressors).

\section{Implications of findings}

Our results have several implications. These data suggest that diabetes prevention and control strategies that do not account for all aspects of social disadvantage may have a limited success. Our findings support the importance of targeting the social environment for 
reducing the risk and burden of diabetes. The development of community-based diabetes prevention translational programs is still in its infancy, ${ }^{32}$ and improving their impact requires more knowledge on aspects of social disadvantage that are important for a cultural adaptation, as well as on the mechanisms by which social disadvantage affects the cost-effectiveness of interventions. Socially disadvantaged populations, especially minorities with limited access to health care, pose unique challenges. They may require investments in terms of time, human capital, and community resources, which would most likely extend beyond the scope of short physician office visits. ${ }^{33}$ The inclusion of a CSR index in diabetes risk assessment tools may improve the identification of at-risk individuals who could be offered targeted enhanced or supplemented care, including longer or more frequent visits, as well as encouraging access to community based interventions designed to reduce the impact of social risks. In the UK, for example, social deprivation has been included in risk assessment tools to identify those at high risk of diabetes; ${ }^{34}$ however, this was based on area level socio-economic status and limited to individual measures of social disadvantage. From a broader policy perspective, the clustering of indicators of social disadvantage in the population indicates the need to address the distal determinants (i.e. upstream social factors) rather than focusing solely on proximal risk factors, especially given that the more distal social risk factors have a greater influence on a range of health conditions. Indeed, effective intervention to reduce disparity in diabetes risk and outcomes may be multilevel strategies targeting individuals, providers, health care organizations, community and health care systems, and health policy.

\section{Strengths and limitations}

The strengths of this study include an examination of a wide range of social risk factors, and their cumulative deleterious effects in a large sample of the US population, accounting for various aspects of social disadvantage which have not been extensively examined previously.

Our study has limitations that merit consideration. First, oral glucose tolerance test was not undertaken; hence the resultant potential misclassification with an underestimation of diabetes frequency biasing our results toward the null. Second, the cross-sectional design of this study does not allow demonstration of a temporal relationship between clustering of social risk factors and diabetes. Thirdly, the cumulative social risk metric assigns equal weights to all risk factors and therefore making the assumption that the risk factors are interchangeable, with components being unweighted. The CSR index assumes that the confluence of social risk factors rather than any single social risk factor is what leads to diabetes. This approach probably simplifies a complex pathway; however, there is no agreement on which social risk factors confer a greater level of risk or whether weighted models outperform unweighted models. However, the CSR models reflect the natural covariation of these social risk factors and it is parsimonious. Another advantage to the CSR model is its simplicity, which makes it readily understandable and communicable to policymakers. Furthermore, we checked our assumption of additivity by examining individual social risk factors and CSR in relation to T2DM simultaneously, which amounted to the attenuation of the CSR effect, suggesting additive rather than synergistic effects of social risk factors. Fourth, single-living, though an easy-to-measure proxy for social isolation and low level of social support, may not be the best of such measures for exploring diabetes as there have been suggestions that living alone is associated with T2DM in men but not women. ${ }^{9}$

Finally, the current proportion of people exposed to social risk factors in the US population may differ from estimates dating back to the study period.

\section{Conclusion}

In summary, we found that an accumulation of social risk factors increased the likelihood of T2DM. This relationship appeared to be mainly driven by the participants' educational levels and ethnicity. The sizable toll that simultaneous exposure to a multitude of social risk factors takes on T2DM risk points toward the need for appropriate clinical and preventive strategies that allocate sufficient and specific resources to address the needs of socially disadvantaged groups.

\section{Declaration of conflicting interests}

The authors declared no potential conflicts of interest with respect to the research, authorship, and/or publication of this article.

\section{Funding}

The authors received no financial support for the research, authorship, and/or publication of this article.

\section{References}

1. Centers for Disease Control. National diabetes statistics report, http://www.cdc.gov/diabetes/pubs/statsreport14/ national-diabetes-report-web.pdf (2014, accessed 22 June 2015).

2. Boyle JP, Thompson TJ, Gregg EW, et al. Projection of the year 2050 burden of diabetes in the US adult population: Dynamic modeling of incidence, mortality, and prediabetes prevalence. Popul Health Metr 2010; 8: 29. 
3. Hill JO, Galloway JM, Goley A, et al. Scientific statement: Socioecological determinants of prediabetes and type 2 diabetes. Diabetes Care 2013; 36: 2430-2439.

4. Agardh E, Allebeck P, Hallqvist J, et al. Type 2 diabetes incidence and socio-economic position: A systematic review and meta-analysis. Int $J$ Epidemiol 2011; 40: 804-818.

5. Sacerdote C, Ricceri F, Rolandsson O, et al. Lower educational level is a predictor of incident type 2 diabetes in European countries: The EPIC-InterAct study. Int $J$ Epidemiol 2012; 41: 1162-1173.

6. Golden SH, Brown A, Cauley JA, et al. Health disparities in endocrine disorders: Biological, clinical, and nonclinical factors - an Endocrine Society scientific statement. J Clin Endocrinol Metab 2012; 97: E1579-E1639.

7. Spanakis EK and Golden SH. Race/ethnic difference in diabetes and diabetic complications. Curr Diab Rep 2013; 13: 814-823.

8. Lidfeldt J, Nerbrand C, Samsioe G, et al. Women living alone have an increased risk to develop diabetes, which is explained mainly by lifestyle factors. Diabetes Care 2005; 28: 2531-2536.

9. Meisinger C, Kandler U and Ladwig K-H. Living alone is associated with an increased risk of type 2 diabetes mellitus in men but not women from the general population: The MONICA/KORA Augsburg Cohort Study. Psychosom Med 2009; 71: 784-788.

10. Murray CJL, Kulkarni SC, Michaud C, et al. Eight Americas: Investigating mortality disparities across races, counties, and race-counties in the United States. PLoS Med 2006; 3: e260.

11. Chichlowska KL, Rose KM, Diez-Roux AV, et al. Life course socioeconomic conditions and metabolic syndrome in adults: The Atherosclerosis Risk in Communities (ARIC) Study. Ann Epidemiol 2009; 19: 875-883.

12. Smith BT, Lynch JW, Fox CS, et al. Life-course socioeconomic position and type 2 diabetes mellitus: The Framingham Offspring Study. Am J Epidemiol 2011; 173: 438-447.

13. Adler NE and Stewart J. Health disparities across the lifespan: Meaning, methods, and mechanisms. Ann NY Acad Sci 2010; 1186: 5-23.

14. Evans GW and Kim P. Multiple risk exposure as a potential explanatory mechanism for the socioeconomic statushealth gradient. Ann N Y Acad Sci 2010; 1186: 174-189.

15. Adler N, Bush NR and Pantell MS. Rigor, vigor, and the study of health disparities. Proc Natl Acad Sci U S A 2012; 109(Suppl.): 17154-17159.

16. Johnson CL, Paulose-Ram R, Ogden CL, et al. National health and nutrition examination survey: analytic guidelines, 1999-2010. Vital Health Stat 2 2013; 161: 1-24.

17. American Diabetes Association. Classification and diagnosis of diabetes. Diabetes Care 2014; 38(Suppl.1): S8-S16.

18. Guenther PM, Reedy J and Krebs-Smith SM. Development of the Healthy Eating Index - 2005. J Am Diet Assoc 2008; 108: 1896-1901.
19. Kraemer HC, Stice E, Kazdin A, et al. How do risk factors work together? Mediators, moderators, and independent, overlapping, and proxy risk factors. Am J Psychiatry 2001; 158: 848-856.

20. Andersen AF, Carson C, Watt HC, et al. Life-course socio-economic position, area deprivation and Type 2 diabetes: Findings from the British Women's Heart and Health Study. Diabet Med 2008; 25: 1462-1468.

21. Maty SC, James SA and Kaplan GA. Life-course socioeconomic position and incidence of diabetes mellitus among blacks and whites: The Alameda County Study, 1965-1999. Am J Public Health 2010; 100: 137-145.

22. Golden SH. A review of the evidence for a neuroendocrine link between stress, depression and diabetes mellitus. Curr Diabetes Rev 2007; 3: 252-259.

23. Greenfield EA and Marks NF. Violence from parents in childhood and obesity in adulthood: Using food in response to stress as a mediator of risk. Soc Sci Med 2009; 68: 791-798.

24. Suglia SF, Staudenmayer J, Cohen S, et al. Cumulative stress and cortisol disruption among Black and Hispanic pregnant women in an urban cohort. Psychol Trauma 2010; 2: 326-334.

25. Champaneri S, Wand GS, Malhotra SS, et al. Biological basis of depression in adults with diabetes. Curr Diab Rep 2010; 10: 396-405.

26. Terry MB, Delgado-Cruzata L, Vin-Raviv N, et al. DNA methylation in white blood cells: Association with risk factors in epidemiologic studies. Epigenetics 2011; 6: 828-837.

27. Waki $\mathrm{H}$, Yamauchi $\mathrm{T}$ and Kadowaki T. The epigenome and its role in diabetes. Curr Diab Rep 2012; 12: 673-685.

28. Slomko H, Heo HJ and Einstein FH. Minireview: Epigenetics of obesity and diabetes in humans. Endocrinology 2012; 153: 1025-1030.

29. Borghol N, Suderman M, McArdle W, et al. Associations with early-life socio-economic position in adult DNA methylation. Int J Epidemiol 2012; 41: 62-74.

30. Subramanyam MA, Diez-Roux AV, Pilsner JR, et al. Social factors and leukocyte DNA methylation of repetitive sequences: The multi-ethnic study of atherosclerosis. PLoS One 2013; 8: e54018.

31. McGuinness D, McGlynn LM, Johnson PCD, et al. Socio-economic status is associated with epigenetic differences in the pSoBid cohort. Int J Epidemiol 2012; 41: 151-160.

32. Ali MK, Echouffo-Tcheugui J and Williamson DF. How effective were lifestyle interventions in real-world settings that were modeled on the Diabetes Prevention Program? Health Aff (Millwood) 2012; 31: 67-75.

33. Fiscella K and Epstein RM. So much to do, so little time: Care for the socially disadvantaged and the 15-minute visit. Arch Intern Med 2008; 168: 1843-1852.

34. Hippisley-Cox J, Coupland C, Robson J, et al. Predicting risk of type 2 diabetes in England and Wales: Prospective derivation and validation of QDScore. BMJ 2009; 338: b880. 